EPJ Web of Conferences 21, 01002 (2012)

DOI: $10.1051 /$ epjconf/20122101002

(C) Owned by the authors, published by EDP Sciences, 2012

\title{
Neutron-induced cross sections of short-lived nuclei via the surrogate reaction method
}

\author{
G. Boutoux ${ }^{1}$, B. Jurado ${ }^{1}$, V. Méot ${ }^{2}$, O. Roig ${ }^{2}$, M. Aïche ${ }^{1}$, L. Mathieu ${ }^{1}$, G. Barreau ${ }^{1}$, N. Capellan ${ }^{1}$, I. \\ Companis $^{1}$, S. Czajkowski ${ }^{1}$, J. T. Burke ${ }^{3}$, E. Bauge ${ }^{2}$, J.M. Daugas ${ }^{2}$, T. Faul ${ }^{2}$, L. Gaudefroy ${ }^{2}$, P.

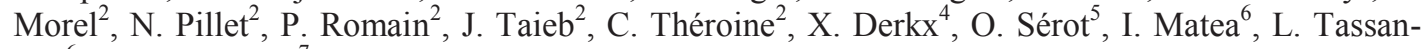 \\ Got $^{6}$ and F. Gunsing ${ }^{7}$ \\ ${ }^{1}$ CENBG, CNRS/IN2P3, Université Bordeaux I, Chemin du Solarium, B.P. 120, 33175 Gradignan, \\ France \\ ${ }^{2}$ CEA DAM DIF, 91297 Arpajon, France \\ ${ }^{3}$ LLNL, Livermore, CA 94550, USA \\ ${ }^{4}$ GANIL, 14076 CAEN, France \\ ${ }^{5}$ CEA-Cadarache, DEN/DER/SPRC/LEPh, 13108 Saint Paul lez Durance, France \\ ${ }^{6}$ IPN, CNRS/IN2P3, Univ. Paris-Sud, 91405 Orsay, France \\ ${ }^{7}$ CEA Saclay, DSM/DAPNIA/SPhN, 91191 Gif-sur-Yvette cedex, France
}

\begin{abstract}
The measurement of neutron-induced cross sections of short-lived nuclei is extremely difficult due to the radioactivity of the samples. The surrogate reaction method is an indirect way of determining cross sections for nuclear reactions that proceed through a compound nucleus. This method presents the advantage that the target material can be stable or less radioactive than the material required for a neutroninduced measurement. We have successfully used the surrogate reaction method to extract neutron-induced fission cross sections of various short-lived actinides. In this work, we investigate whether this technique can be used to determine neutron-induced capture cross sections in the rare-earth region.
\end{abstract}

\section{Introduction}

Neutron-induced radiative-capture cross sections of short-lived nuclei are crucial for fundamental nuclear physics and also for applications such as reactor physics and astrophysics. In particular, these data are needed to test s- and r-process models. The latter are used to understand the synthesis of the elements between iron and uranium in astrophysical environments. However, very often the high radioactivity of the samples makes the direct measurement of these cross sections extremely difficult. The surrogate reaction method is an indirect way of determining cross sections for compound nuclear reactions. This method was first proposed by J.D. Cramer and H.C. Britt [1] in the seventies and is schematically represented in figure 1 . The left part of figure 1 illustrates a neutron-induced reaction on target $A$-1, which leads to the compound-nucleus $A$ at an excitation energy $E^{*}$. The nucleus $A^{*}$ can decay through different exit channels: fission, gamma-decay, neutron emission, etc... On the right part of figure 1, in the surrogate reaction method, the same compound nucleus $A^{*}$ is produced by a transfer reaction between a projectile $y$ (a light charged particle) and a target $X$. The transfer reaction $(y+X \rightarrow A+w)$ leads to a heavy recoil nucleus $A^{*}$ and an ejectile $w$. The

This is an Open Access article distributed under the terms of the Creative Commons Attribution-Noncommercial License 3.0, which permits unrestricted use, distribution, and reproduction in any noncommercial medium, provided the original work is properly cited. 
identification of the ejectile permits to determine the mass $A$ and charge $Z$ of the decaying nucleus. In addition, we can deduce the excitation energy $E^{*}$ of the compound nucleus $A$ by measuring the kinetic energy and the emission angle of the ejectile $w$. The measurement of the number of coincidences between the ejectiles and the decay products normalised to the total number of detected ejectiles allows one to extract the decay probability $P_{\text {decay }}^{A \text {,exp }}$ for the corresponding decay channel. According to the surrogate reaction method, the neutron-induced cross section for the nucleus $A-1$ is then given by the equation:

$$
\sigma_{\text {decay }}^{A-1}\left(E_{n}\right) \cong \sigma_{C N}^{A}\left(E_{n}\right) \cdot P_{\text {decay }}^{A, \exp }\left(E^{*}\right)
$$

where $\sigma_{C N}^{A}$ is the calculated compound nuclear formation cross section in the desired reaction (formation of the nucleus $A$ after a neutron absorption with an energy $E_{n}$ ). In our case, $\sigma_{C N}^{A}$ is obtained from optical model calculations performed with the code TALYS [2]. The relation between incident neutron energy $E_{n}$ and excitation energy $E^{*}$ of the compound nucleus $A$ can be written as:

$$
E^{*}=S_{n}+E_{n} \cdot \frac{A-1}{A}
$$

where $S_{n}$ is the one-neutron separation energy in the nucleus $A$. The interest of this method is that in some cases the target $X$ is stable or less radioactive than the target $A-1$.

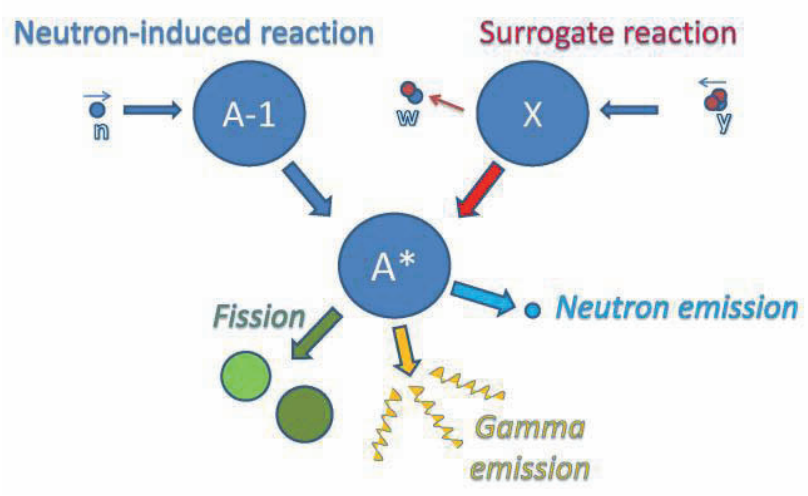

Fig. 1: Schematic representation of the surrogate reaction method. The surrogate reaction is here a transfer reaction $X(y, w) A^{*}$. Three possible exit channels (fission, gamma emission and neutron emission) are also represented.

Recently, we used the surrogate reaction method to determine the neutron-induced fission cross sections of ${ }^{242} \mathrm{Cm}\left(T_{1 / 2}=162.8 \mathrm{~d}\right),{ }^{243} \mathrm{Cm}\left(T_{1 / 2}=29.1 \mathrm{y}\right)$ and ${ }^{241} \mathrm{Am}\left(T_{1 / 2}=432.2 \mathrm{y}\right)$. To reach and study these nuclei we employed few-nucleon transfer reactions using a ${ }^{3} \mathrm{He}$ projectile on a ${ }^{243} \mathrm{Am}$ $\left(T_{1 / 2}=7370 \mathrm{y}\right)$ target. All details are given in [3]. A remarkable good agreement was observed between our results and the existing neutron-induced data at the lowest neutron energies. In the present work, we investigate whether the surrogate reaction technique can be used to infer neutroninduced capture cross sections. 


\section{$\mathrm{CNR} * 11$}

\section{Validity of the surrogate reaction method}

The neutron-induced reaction and the transfer reaction permit to produce the same compound nucleus in $Z, A$ and $E^{*}$. However, the angular momentum $(J)$ and parity $(\pi)$ distributions populated by a transfer reaction may not be the same as the ones populated in a neutron-induced reaction. Since at low $E^{*}$ the decay probabilities may strongly depend on $J^{\pi}$, the decay probability obtained in surrogate experiments can be very different from the one measured in neutron-induced experiments.

Assuming that the nucleus $A^{*}$ is in a compound state, its formation and decay are independent and the decay probabilities are given by:

$$
\begin{aligned}
& P_{n}^{\text {decay }}\left(E^{*}\right)=\sum_{J^{\pi}} F_{n}\left(E^{*}, J^{\pi}\right) \cdot G_{\text {decay }}\left(E^{*}, J^{\pi}\right) \\
& P_{t}^{\text {decay }}\left(E^{*}\right)=\sum_{J^{\pi}} F_{t}\left(E^{*}, J^{\pi}\right) \cdot G_{\text {decay }}\left(E^{*}, J^{\pi}\right)
\end{aligned}
$$

where the indices $n$ and $t$ stand for neutron and transfer reactions, respectively, $F_{n}\left(E^{*}, J^{\pi}\right)$ and $F_{t}\left(E^{*}, J^{\pi}\right)$ correspond to the probability that the compound nucleus is formed in the state $J^{\pi}$ by the neutron-induced and the transfer reaction, respectively. $G_{\text {decay }}\left(E^{*}, J^{\pi}\right)$ is the branching ratio for a given decay channel. The two decay probabilities of eqs. (3) and (4) are equal in two cases:

1) The $J^{\pi}$ distributions populated in both reactions are similar:

$$
F_{n}\left(E^{*}, J^{\pi}\right) \approx F_{t}\left(E^{*}, J^{\pi}\right)
$$

Unfortunately, the experimental and/or theoretical determination of the angular momentum populated in transfer reactions represents a big challenge. An important effort from theoreticians and experimentalists should be done in order to determine these distributions. We will see below how the present work can provide very valuable information on this issue.

2) The branching ratios are independent of $J^{\pi}$ :

$$
G_{\text {decay }}\left(E^{*}, J^{\pi}\right)=G_{\text {decay }}\left(E^{*}\right)
$$

Then the branching ratios can be taken out of the summation signs in eqs. (3) and (4). Since

$$
\sum_{J^{\pi}} F_{n}\left(E^{*}, J^{\pi}\right)=1
$$

$P_{n}^{\text {decay }}\left(E^{*}\right) \approx P_{t}^{\text {decay }}\left(E^{*}\right)$ and the cross section for the desired reaction takes the simple product form of eq. (1). This second hypothesis is known as the Weisskopf-Ewing approximation [4] and is justified for high excitation energies where the decay of the compound-nucleus is dominated by statistical level densities. At lower excitation energies, the decay probabilities strongly depend of the $J^{\pi}$ of discrete states, whose population depends on the reaction mechanism used to produce the compound nucleus $A^{*}$. 
In Ref. [3] we showed that our results for the fission cross sections obtained with the surrogate method are in very good agreement with the neutron-induced data at low excitation energies. The reason is that for all the reactions considered in [3] the excitation energy of the decaying nuclei was high enough for the Weisskopf-Ewing approximation to be valid. In radiative capture reactions, however, we have to consider the competition with neutron emission to the ground or first excited states which is much more sensitive to the differences between the spin-parity distributions [5]. In addition, in the case of actinides, one may need to distinguish between gamma rays originating from the fission fragments and radiative capture gamma rays. This can make radiative capture measurements extremely complicated. Therefore, as a first step we have chosen to investigate the validity of the surrogate method for radiative capture reactions on rare earth nuclei. In particular, our aim is to study the transfer reactions ${ }^{174} \mathrm{Yb}\left({ }^{3} \mathrm{He}, \mathrm{p} \gamma\right){ }^{176} \mathrm{Lu}$ and ${ }^{174} \mathrm{Yb}\left({ }^{3} \mathrm{He},{ }^{4} \mathrm{He} \gamma\right){ }^{173} \mathrm{Yb}$ as surrogate for the ${ }^{175} \mathrm{Lu}(\mathrm{n}, \gamma)$ and ${ }^{172} \mathrm{Yb}(\mathrm{n}, \gamma)$ reactions, respectively. We have considered the ${ }^{175} \mathrm{Lu}(\mathrm{n}, \gamma)$ and ${ }^{172} \mathrm{Yb}(\mathrm{n}, \gamma)$ cross sections because they present the advantage to be very well known, see for example $[6,7,8,9]$.

\section{Experimental set-up}

The measurement was performed at the Tandem accelerator of the IPN Orsay. We used an incident ${ }^{3} \mathrm{He}$ beam with an energy of $24 \mathrm{MeV}$. The beam intensity was 20 particle $\mathrm{nA}$. The stable ${ }^{174} \mathrm{Yb}$ target was fabricated at the SIDONIE facility of the CSNSM laboratory. The ${ }^{174} \mathrm{Yb}$ sample had a thickness of $250 \mu \mathrm{g} / \mathrm{cm}^{2}$ and was deposited onto a C foil of $50 \mu \mathrm{g} / \mathrm{cm}^{2}$. Figure 2 illustrates our experimental set-up. To infer the radiative capture probability, gamma rays were detected in coincidence with the ejectiles. The latter were fully identified by two large area $\Delta \mathrm{E}-\mathrm{E}$ telescopes placed symmetrically at $130^{\circ}$ with regard to the ${ }^{3} \mathrm{He}$ beam. The $\Delta \mathrm{E}$ detectors were two $300 \mu \mathrm{m}$ silicon position sensitive detectors, whose $16^{*} 16 \mathrm{X}-\mathrm{Y}$ strips provided the angle of the detected particle with an angular coverage of $108^{\circ}$ to $152^{\circ}$. The E detectors were two $\mathrm{Si}(\mathrm{Li})$ detectors of $3 \mathrm{~mm}$ thickness. We shielded the Si detectors against delta electrons coming from the target with a thin Mylar(Al) foil polarized at $-300 \mathrm{~V}$.

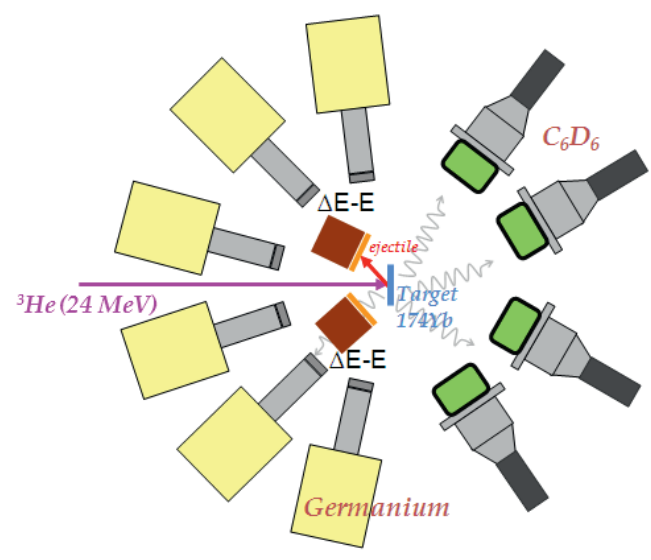

Fig. 2 : Top view of the experimental set-up for radiative capture probability measurements. The four $\mathrm{C}_{6} \mathrm{D}_{6}$ liquid scintillators were placed at forward angles with respect to the beam direction, whereas the two Si telescopes and the six germanium detectors where placed at backward angles.

Four $\mathrm{C}_{6} \mathrm{D}_{6}$ liquid scintillators were used for gamma detection to infer the radiative capture probability. The use of $\mathrm{C}_{6} \mathrm{D}_{6}$ liquid scintillators has the important advantage that the coupling of these detectors to a pulse shape discriminator permits to separate between photons and neutrons interacting within the scintillators. The ${ }^{174} \mathrm{Yb}$ target was also surrounded by six high-volume germanium detectors. They were used to measure low-lying $\gamma$-ray transition intensities as a function 


\section{$\mathrm{CNR} * 11$}

of the compound-nucleus excitation energy, which is an additional way to investigate the radiative capture probability and the difference in spin distributions between transfer and neutron-induced reactions.

The ${ }^{3} \mathrm{He}$-induced transfer reactions on the ${ }^{174} \mathrm{Yb}$ target lead to the production of various heavy residues. In this work we consider only the $\left({ }^{3} \mathrm{He}, \mathrm{p}\right)$ and the $\left({ }^{3} \mathrm{He},{ }^{4} \mathrm{He}\right)$ channels because they lead to nuclei for which there exist neutron-induce data to compare with. The advantage of using transfer reactions is clear: the simultaneous access to two transfer channels allowed us to study two nuclei from a single projectile-target combination. Moreover, since there are two bodies in the outgoing reaction channel, the excitation energy of the decaying nucleus $E^{*}$ follows a broad probability distribution.

\section{Results}

The experimental radiative capture probability $P \gamma\left(E^{*}\right)$ can be obtained in the following way:

$$
P_{\gamma}\left(E^{*}\right)=\frac{N_{\text {coinc }}\left(E^{*}\right)}{N_{\text {single }}\left(E^{*}\right)} \cdot \frac{1}{\varepsilon\left(E^{*}\right)}
$$

where $N_{\text {coinc }}\left(E^{*}\right)$ is the number of ejectiles detected in coincidence with the $\mathrm{C}_{6} \mathrm{D}_{6}$ detectors, $N_{\text {singles }}\left(E^{*}\right)$ the total number of ejectiles, i.e. the total number of decaying nuclei formed, and $\varepsilon\left(E^{*}\right)$ represents the $\mathrm{C}_{6} \mathrm{D}_{6}$ cascade detection efficiency.

\subsection{Results for the ${ }^{174} \mathrm{Yb}\left({ }^{3} \mathrm{He}, \mathrm{p}\right){ }^{176} \mathrm{Lu}$ * reaction}

For this transfer channel the $N_{\text {singles }}\left(E^{*}\right)$ and $N_{\text {coinc }}\left(E^{*}\right)$ spectra had to be corrected for the ejectiles coming from transfer reactions between the ${ }^{3} \mathrm{He}$ beam and the carbon backing. Since the ${ }^{176} \mathrm{Lu}$ is formed by a transfer reaction, it is possible to extend our investigation below the neutron separation energy $S_{n}$ where only $\gamma$-rays can be emitted and consequently the measured radiative capture probability should be 1 . The probability to detect with our set-up various gamma rays coming from the same cascade is less than $3.8 \%$. Therefore, below $S_{n}$ the ratio $N_{\text {coinc }}\left(E^{*}\right) / N_{\text {singles }}\left(E^{*}\right)$ gives the total efficiency of the $\mathrm{C}_{6} \mathrm{D}_{6}$ detectors for detecting a gamma-cascade. Applying a threshold to the gammaenergy in order to suppress the contribution of the $\left({ }^{3} \mathrm{He}, \mathrm{pn} \gamma\right)^{175} \mathrm{Lu}^{*}$ channel, this ratio remains essentially constant from $E^{*}=5.5 \mathrm{MeV}$ to $S_{n}$, see figure 3 . The constancy of this ratio is to be expected as TALYS calculations show that the multiplicity of the gamma cascade and the average gamma energy vary only very weakly between $S_{n}$ and $8 \mathrm{MeV}$ excitation energy. Thus, it is reasonable to assume a constant efficiency in the vicinity of $S_{n}$. The independence of the cascade detection efficiency with the excitation energy and its absolute value have been confirmed by using the total-energy detection principle in combination with the pulse-height weighting technique. This technique allows one to extract the cascade detection efficiency independently from the measured data. It was used in previous surrogate studies using the ${ }^{232} \mathrm{Th}\left({ }^{3} \mathrm{He}, \mathrm{p}\right)$ reaction $[10,11]$. Once the detection efficiency is determined, we apply equation (8) to determine the radiative capture probability of ${ }^{176} \mathrm{Lu}$ as a function of $E^{*}$, see figure 3. Our results are compared with TALYS calculations for the neutron-induced capture probability of ${ }^{175} \mathrm{Lu}$ and for the gamma-induced capture reaction of ${ }^{176} \mathrm{Lu}$. The parameters of the TALYS code have been tuned to reproduce the experimental data for the ${ }^{175} \mathrm{Lu}(\mathrm{n}, \gamma)$ cross sections. The results of figure 3 show that our surrogate data present big discrepancies with respect to the neutron-induced data, while we observe a very good agreement with the ${ }^{176} \mathrm{Lu}(\gamma, \gamma)$ calculation at low energies. This indicates that the $J^{\pi}$ distribution populated in the $\left({ }^{3} \mathrm{He}, \mathrm{p}\right)$ surrogate reaction is close to the one populated in the photon-induced reaction. The ground state $J^{\pi}$ of ${ }^{175} \mathrm{Lu}$ and ${ }^{176} \mathrm{Lu}$ are respectively $7 / 2^{+}$and $7^{-}$. Therefore, the angular momentum of ${ }^{176} \mathrm{Lu}$ populated by low energy neutrons is mainly centered between 3 and $4 \hbar$, while the angular 
momentum populated in the gamma-induced reaction is centered around 6,7 and $8 \mathrm{~h}$. Consequently, the big discrepancies found at low $E^{*}$ can be explained by the differences between the spin distributions populated in transfer and neutron-induced reactions. For excitation energies after neutron emission below the first excited state of ${ }^{175} \mathrm{Lu}\left(113 \mathrm{keV}, 9 / 2^{+}\right)$, the $(\mathrm{n}, \gamma)$ decay channel is only in competition with the compound-elastic channel $(n, n)$, where the residual nucleus is left in its ground state after neutron emission. Since the nucleus can only decay to one state with a welldefined $J^{\pi}(7 / 2+)$ and the angular momentum carried by the emitted neutron is very small, this particular exit channel is extremely sensitive to the spin of the compound nucleus ${ }^{176} \mathrm{Lu}^{*}$. TALYS calculations show that the $(n, n)$ channel remains rather strong up to about $7 \mathrm{MeV}$. As said above, the angular momentum induced by the $\left({ }^{3} \mathrm{He}, \mathrm{p}\right)$ transfer reaction seems to be also centered around 7 or 8 $\hbar$, which is about two times higher than the angular momentum of the ${ }^{175} \mathrm{Lu}$ ground state $7 / 2$, leading to a suppression of the compound-elastic channel. The same strong spin selectivity is also expected for the first excited states of the residual nucleus. Therefore, at the lowest energies above $S_{n}$, gamma emission is the dominant decay channel. In conclusion, the differences in populated spins and the high selectivity of the neutron decay channel are at the origin of the large discrepancies observed between surrogate and neutron-induced measurements.

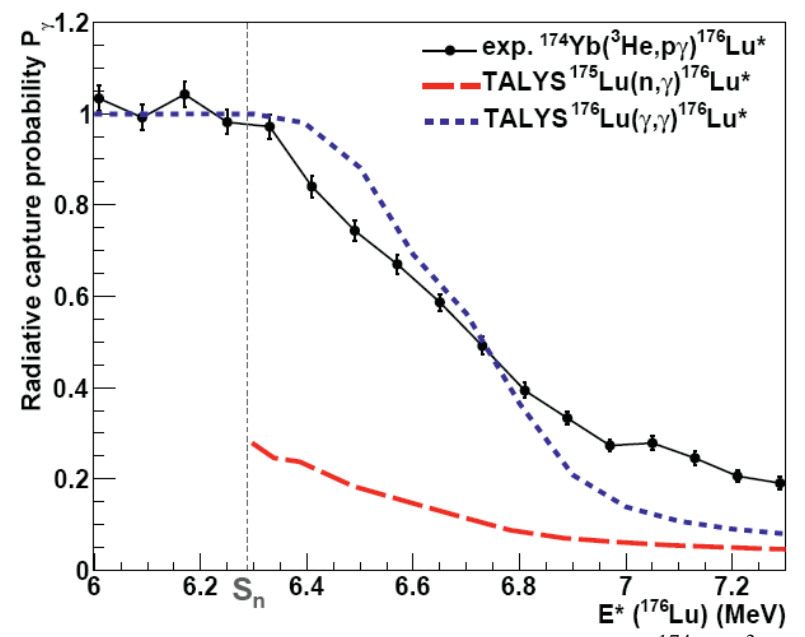

Fig. 3 : Results for the radiative capture probability measured in the ${ }^{174} \mathrm{Yb}\left({ }^{3} \mathrm{He}, \mathrm{p}\right){ }^{176} \mathrm{Lu}$ reaction. The red line is the result of a calculation performed with TALYS for the neutron-induced capture probability of ${ }^{176} \mathrm{Lu}$. The blue line is TALYS result for the photon-induced capture probability of ${ }^{176} \mathrm{Lu}$.

Since the gamma decay probability of ${ }^{176} \mathrm{Lu}$ is very sensitive to $J^{\pi}$, we investigated a method to extract the populated $J^{\pi}$ distribution from a fit to the experimental decay probability using the branching ratios calculated by TALYS. Figure 4 shows TALYS calculations for the gamma-decay probabilities for various spin/parity states as a function of the excitation energy of ${ }^{176}$ Lu. Figure 4 clearly illustrates the sensitivity of gamma-decay probabilities to the $J^{\pi}$ of the decaying compound state. The clear drop just above $S_{n}$ due to the competition with the $(n, n)$ channel is only observed for spin values of 3 to $4 \hbar$. For all the other spins $\mathrm{P}_{\gamma}$ remains close to 1 near $S_{n}$. Note also that for the highest spins, $\mathrm{P}_{\gamma}$ remains equal to 1 over several $\mathrm{MeV}$. This is due to the impossibility to populate the first excited states of ${ }^{175} \mathrm{Lu}$ after neutron emission. At these excitation energies the emitted neutron will most probably carry small angular momentum. It is clear that the Weisskopf-Ewing approximation is not valid in this region. 

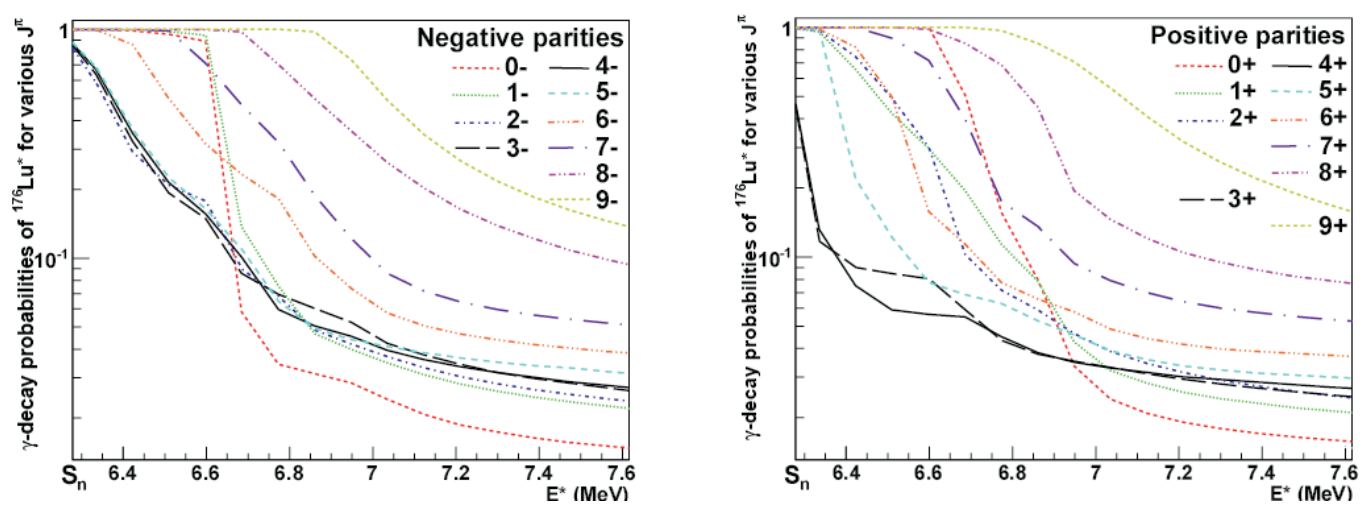

Fig. 4: Calculated branching-ratios $G_{\gamma}\left(E^{*}, J^{\pi}\right)$ of ${ }^{176} \mathrm{Lu}$. The excitation energies shown correspond to incident neutron energies of $0-1,2 \mathrm{MeV}$. The left and right parts are dedicated to negative and positive parities, respectively.

According to equation (4), the experimental gamma-decay probability $P_{\gamma}\left(E^{*}\right)$ can be written as:

$$
P_{\gamma}\left(E^{*}\right)=\sum_{J^{\pi}}\left\lfloor\frac{1}{2 \sigma \sqrt{2 \pi}} e^{-\frac{(J-\bar{J})^{2}}{2 \sigma^{2}}}\right\rfloor \cdot G_{\gamma}\left(E^{*}, J^{\pi}\right)
$$

where the angular momentum distribution $F_{t}\left(J^{\pi}\right)$ is expressed as a Gaussian without dependence on the excitation energy. The two parities are assumed to be equally populated. The two unknown parameters $\bar{J}$ and $\sigma$ correspond to the average value and the standard deviation of the spin distribution. These quantities are obtained by fitting eq. (9) to the experimental radiative capture probability using the branching ratios $G_{\gamma}\left(E^{*}, J^{\pi}\right)$. By fitting our data from 5.5 to $7.4 \mathrm{MeV}$, as shown in figure 5 , we obtained a spin distribution centered at $\bar{J}=7.1 \hbar$ with $\sigma=2.3 \hbar$. For comparison, the spin distribution populated in the neutron-induced reaction for $E_{n}=1 \mathrm{MeV}$ has $\bar{J} \approx$ $4 \hbar$ with $\sigma=1.3 \hbar$.

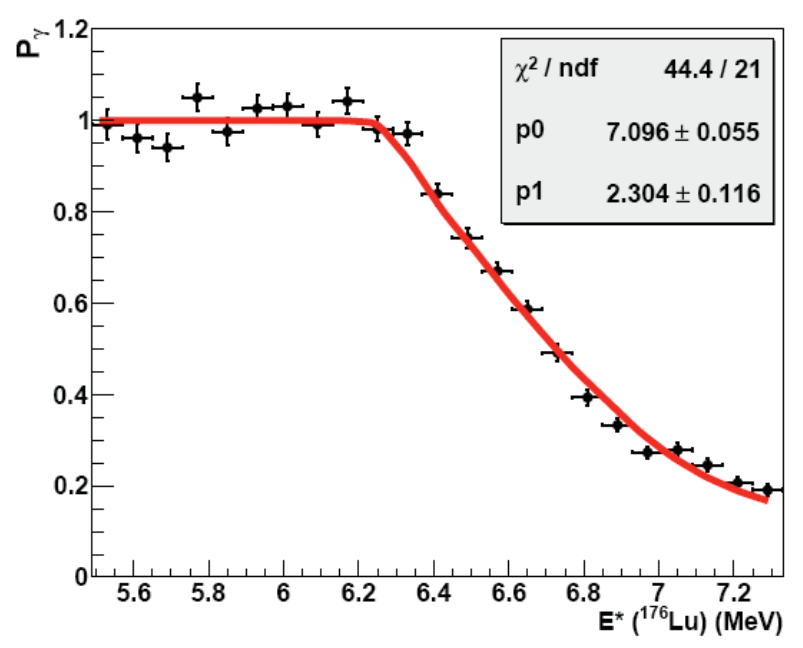


Fig. 5 : Fit of the experimental $\mathrm{P}_{\gamma}\left({ }^{176} \mathrm{Lu} \mathrm{u}^{*}\right)$ with eq. (9). The parameters $p 0$ and $p 1$ correspond to the average spin-value $\bar{J}$ and the $\sigma$ of the Gaussian spin distribution, respectively.

We would like to stress that we also investigated a formalism to extract the transferred angular momentum and spin distributions through a more realistic approach that does not assume equal probability for positive and negative parities. We follow the fairly simple statistical assumptions already introduced by B. Back [12] and W. Younes [13]. This work is not detailed in this contribution but it confirms the latter spin distribution.

Our previous conclusion is reinforced by the measurement of low-lying $\gamma$-ray transition intensities performed with the germanium detectors. Figure 6 shows the Germanium gamma-ray spectrum in coincidence with protons for various excitations energies. The gamma-ray transitions coming from de-excitation of the $8^{+}$level of ${ }^{176} \mathrm{Lu}$, located at $424.9 \mathrm{keV}$, are clearly observed at $424.9 \mathrm{keV}$ and $241 \mathrm{keV}$. In the ${ }^{175} \mathrm{Lu}(\mathrm{n}, \gamma)$ reaction at thermal neutron energies [14] these gamma-ray transitions are observed with an intensity 10 times lower than the $139.3 \mathrm{keV}$ transition coming form the $4^{+}$level $\left(E^{*}=372.5 \mathrm{keV}\right)$, which is not observed in our surrogate data. For $1 \mathrm{MeV}$ neutron energy, the gamma-ray intensity ratio $(139.3 \mathrm{keV} / 424.9 \mathrm{keV})$ is predicted to be around 4 in our $(\mathrm{n}, \gamma)$ TALYS calculation. The comparison of the three spectra shown in figure 6 reflects very clearly the contamination due to the decay of ${ }^{175} \mathrm{Lu}$ produced in the reaction $\left({ }^{3} \mathrm{He}, \mathrm{pn} \gamma\right)$. The bottom panel of figure 6 shows that above $E^{*}=6.9 \mathrm{MeV}$ the decay is dominated by this channel. As mentioned before, a threshold of $200 \mathrm{keV}$ and $400 \mathrm{keV}$ in the gamma energy measured by the $\mathrm{C}_{6} \mathrm{D}_{6}$ is set for $\mathrm{E}^{*}=S_{n}+600 \mathrm{keV}$ and above this limit, respectively, to remove the contribution of the $\left({ }^{3} \mathrm{He}, \mathrm{pn} \gamma\right)$ reaction. These values of the threshold come from the fact that for ${ }^{175} \mathrm{Lu}$ all the gamma-ray energies that have been measured between $E^{*}=S_{n}$ and $S_{n}+600 \mathrm{keV}$ and between $S_{n}+600 \mathrm{keV}$ and $S_{n}+1$ $\mathrm{MeV}$ are below $200 \mathrm{keV}$ and $400 \mathrm{keV}$, respectively.
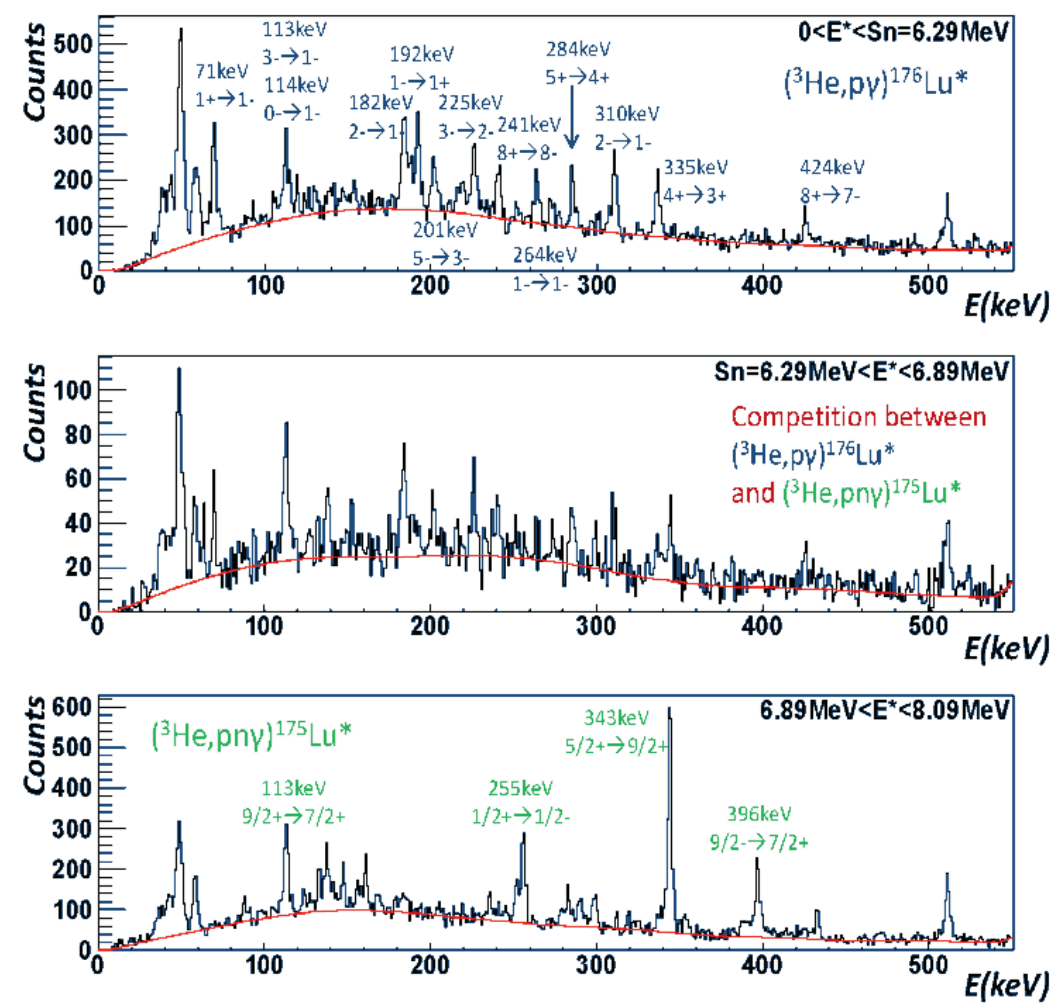


\section{CNR*11}

Fig. 6 : Germanium gamma-ray spectra for three ranges of $E^{*}$. One can identify the main $\gamma$-ray transitions related to the $\left({ }^{3} \mathrm{He}, \mathrm{p} \gamma\right){ }^{176} \mathrm{Lu}^{*}$ and $\left({ }^{3} \mathrm{He}, \mathrm{pn} \gamma\right){ }^{175} \mathrm{Lu}^{*}$ reactions. The background is shown in red.

\subsection{Results for the ${ }^{174} \mathrm{Yb}\left({ }^{3} \mathrm{He},{ }^{4} \mathrm{He}\right){ }^{173} \mathrm{Yb}^{*}$ reaction}

The same analysis as the one presented in the previous section was performed to extract the radiative capture probability associated to the ${ }^{174} \mathrm{Yb}\left({ }^{3} \mathrm{He},{ }^{4} \mathrm{He}\right)^{173} \mathrm{Yb}$ reaction. A similar gamma-energy threshold for the $\mathrm{C}_{6} \mathrm{D}_{6}$ detectors was necessary to remove the contribution from the ${ }^{174} \mathrm{Yb}\left({ }^{3} \mathrm{He}\right.$, ${ }^{4} \mathrm{Hen} \gamma$ ) reaction. The results for the gamma decay probability are shown in figure 7 . The data are compared with TALYS results for the neutron and photon-induced radiative capture probabilities. The parameters of the code have been fixed to best reproduce the existing neutron-induced data. Again, the transfer-induced results are much higher (by a factor 10 at the lowest energies!) than the neutron-induced data. In this case, the photon-induced capture probability is also bellow the transferinduced data. The two TALYS calculations show clear changes of slope at $S_{n}$ and at energies that correspond to the first and second excited state of ${ }^{172} \mathrm{Yb}$. They indicate the reduction of the gamma decay probability caused by the competition with neutron emission leaving the residual nucleus ${ }^{172} \mathrm{Yb}$ in the ground state, in the first or the second excited state. These changes in slope can also be observed at similar energies for the ${ }^{174} \mathrm{Yb}\left({ }^{3} \mathrm{He},{ }^{4} \mathrm{He}\right){ }^{173} \mathrm{Yb}$ reaction although in this case the changes due to higher states are also observed. Note that the excitation-energy resolution for these data is 80 $\mathrm{keV}$. Also the drops in the radiative capture probability are less intense than for the $(\mathrm{n}, \gamma)$ and $(\gamma, \gamma)$ reactions. This suggests that the angular momentum populated in the transfer reaction is significantly bigger than the one populated in the neutron and photon-induced reactions. The ground state of ${ }^{173} \mathrm{Yb}$ is $5 / 2$, therefore the angular momentum of ${ }^{173} \mathrm{Yb}$ after photon absorption will be centered around $3 / 2$ and $7 / 2$. These values are smaller than the ones populated for ${ }^{176} \mathrm{Lu}$ whose ground-state spin is $7^{+}$. This explains why in this case the photon-induced reaction is clearly below the transfer reaction.

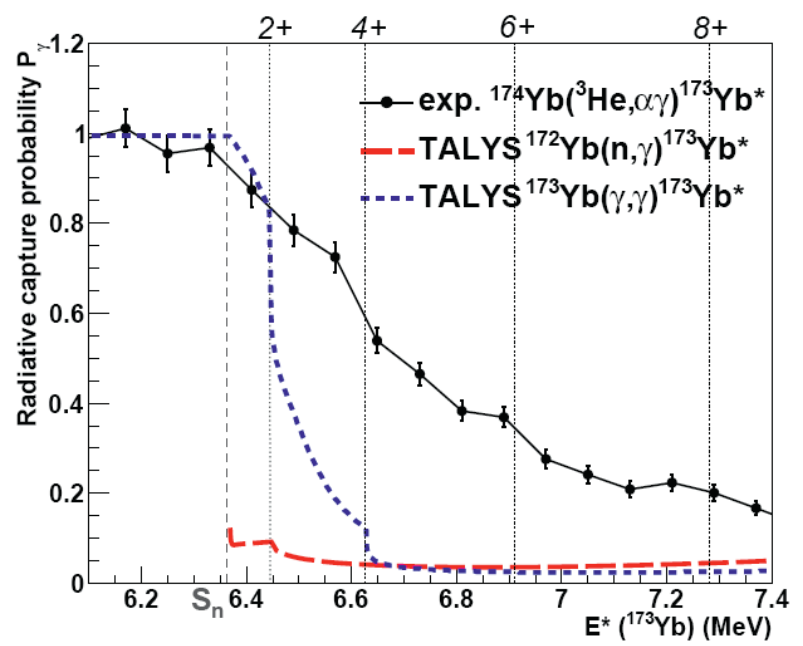

Fig. 7: Results for the radiative capture probability measured in the ${ }^{174} \mathrm{Yb}\left({ }^{3} \mathrm{He},{ }^{4} \mathrm{He}\right){ }^{173} \mathrm{Yb}$ reaction. The red line is the result of a calculation performed with TALYS for the neutron-induced capture probability of ${ }^{173} \mathrm{Yb}$. The blue line is TALYS result for the photon-induced capture probability of ${ }^{173} \mathrm{Yb}$. The arrows indicate the position of the first excited states of ${ }^{172} \mathrm{Yb}$. 

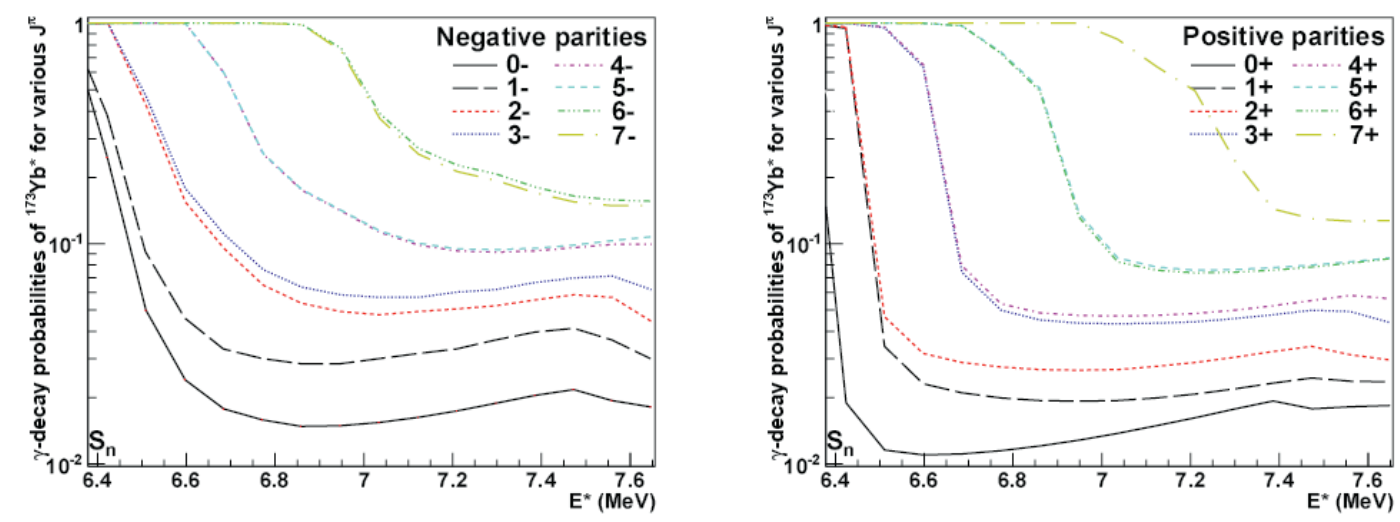

Fig. 8: Calculated branching-ratios $G_{\gamma}\left(E^{*}, J^{\pi}\right)$ of ${ }^{173} \mathrm{Yb}$. The excitation energies shown correspond to incident neutron energies of $0-1,2 \mathrm{MeV}$. The left and right parts are dedicated to negative and positive parities, respectively.

Figure 8 shows TALYS calculations of the gamma branching ratios as a function of the excitation energy of the ${ }^{173} \mathrm{Yb}$ compound-nucleus. Due to the low level density in the residual even-even nucleus ${ }^{172} \mathrm{Yb}$ below the pairing gap, the onset of the decay to of each state corresponds clearly to a discontinuity. The drop at $S_{n}$ corresponds to the opening of the elastic neutron channel that is only observed for spin values close to $0 \mathrm{~h}$ and is particularly strong for positive parity. Therefore, the Weisskopf-Ewing approximation is clearly not appropriate. As a conclusion, gamma-decay probabilities are extremely sensible to $J^{\pi}$ because of the high spin-selectivity of neutron-decay. Gamma-decay is therefore strongly influenced by the structure of the low-lying states of the residual nucleus after neutron emission. As for the $\left({ }^{3} \mathrm{He}, \mathrm{p}\right)$ reaction, we can extract rather direct information on the transferred angular momentum in the $\left({ }^{3} \mathrm{He},{ }^{4} \mathrm{He}\right)$ reaction from a fit to the experimental capture probability using equation (9) and the branching ratios from TALYS. As illustrated in figure 9, we obtained a spin distribution centered on $\bar{J}=3.9 \hbar$ with $\sigma=3.2 \hbar$. The considered energy range was extended from 5.5 to $7.5 \mathrm{MeV}$. For comparison, the spin distribution populated in a neutron-induced reaction for $E_{n}=1 \mathrm{MeV}$ has $\bar{J}=0 \mathrm{~h}$ with $\sigma=1.3 \mathrm{~h}$. We think this study provides valuable information on the angular momentum transferred in the $\left({ }^{3} \mathrm{He},{ }^{4} \mathrm{He}\right)$ reaction.

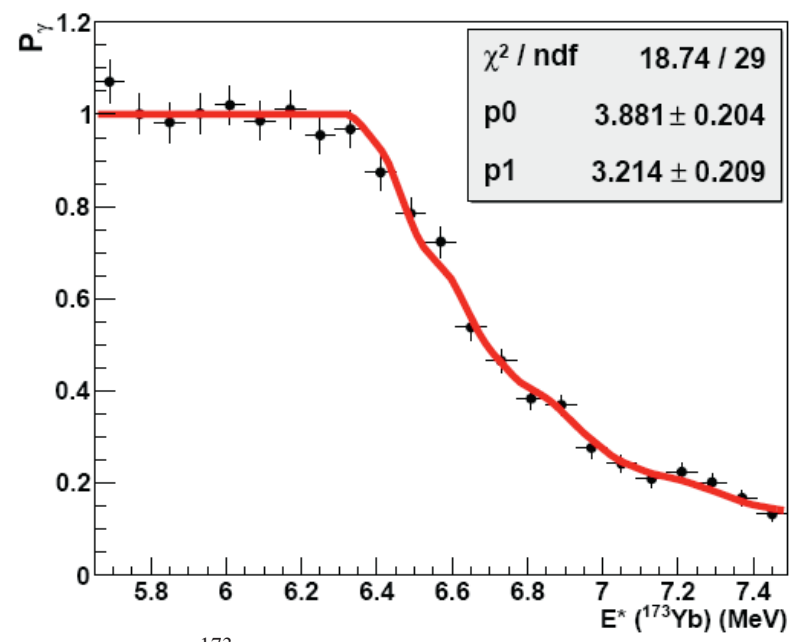

Fig. 9 : Fit of the experimental $\mathrm{P}_{\gamma}\left({ }^{173} \mathrm{Yb}^{*}\right)$ with eq. 9. The parameters $p 0$ and $p 1$ correspond to the average spin-value $\bar{J}$ and the $\sigma$ of the Gaussian spin distribution, respectively. 


\section{Conclusion}

We have performed an experiment to study the validity of the surrogate method for extracting neutron-induced capture cross sections. We have used the well known ${ }^{175} \mathrm{Lu}(\mathrm{n}, \gamma)$ and ${ }^{172} \mathrm{Yb}(\mathrm{n}, \gamma)$ cross sections to study the ${ }^{174} \mathrm{Yb}\left({ }^{3} \mathrm{He}, \mathrm{p}\right){ }^{176} \mathrm{Lu}^{*}$ and ${ }^{174} \mathrm{Yb}\left({ }^{3} \mathrm{He},{ }^{4} \mathrm{He}\right){ }^{173} \mathrm{Yb}$ surrogate reactions. Our surrogate data present big discrepancies with respect to the neutron-induced data. Since the gammadecay probabilities are very sensitive to $J^{\pi}$, we have extracted the populated angular-momentum distributions from a fit to the experimental decay probability using the branching ratios calculated by TALYS. The average spins populated in these transfer reactions are found to be a factor 2 to 4 higher than the ones populated in the neutron-induced reactions. Right above $S_{n}$, neutron emission to the ground state of the residual nucleus is the dominant way of deexcitation for a neutron-induced reaction, whereas in the transfer reactions used, this type of decay is highly improbable and consequently gamma-decay is favoured. One expects that neutron emission to the first excited states is also considerably suppressed for the same reasons. The Weisskopf-Ewing approximation cannot be applied at low neutron energies. The sensitivity of the decay probability to $J^{\pi}$ decreases as the number of states in the residual nucleus after neutron evaporation increases. For this reason, the case of actinides is expected to be better [15]. We believe that an important effort from theoreticians and experimentalists needs to be done in order to determine the angular momentum distributions populated in the surrogate reactions. An important point to be investigated is the dependence of the transferred angular-momentum distribution on the target nucleus. It should be investigated to which extent the obtained angular momentum distribution can be extrapolated to heavier target nuclei (e.g. actinides). More precisely, one needs to study the influence of the single-particle structure of the target nucleus on the angular momentum distribution. If progress is made on this issue, we could use decay probabilities measured with the surrogate method together with experimental or theoretical spin distributions to fix key parameters of the statistical model. The latter can then be used in combination with the optical model to provide reliable predictions of neutron-induced cross sections for unstable nuclei that cannot be measured. In this sense, the surrogate method in combination with radioactive ion beams (RIB) can help explore regions of the chart of nuclei that cannot be studied with surrogate reactions using direct kinematics. The $(d, p)$ reaction is particularly interesting for future experiments in inverse kinematics. That is why, in the near future we plan to investigate the ${ }^{238} \mathrm{U}(\mathrm{d}, \mathrm{p})$ reaction as surrogate for the ${ }^{238} \mathrm{U}(\mathrm{n}, \mathrm{f})$ and ${ }^{238} \mathrm{U}(\mathrm{n}, \gamma)$ reactions. Very interesting opportunities for surrogate studies in inverse kinematics open up with new RIB facilities such as HIE-ISOLDE or SPIRAL2. In the very long term, unprecedented surrogate experiments on fission will become possible thanks to the ELISE $e^{-}$-ion collider [16]. The fissioning nucleus will be fully characterized in $\left(A, Z, E^{*}, J, \pi\right)$ and a complete set of fission observables will be precisely measured as a function of $E^{*}$.

\section{Acknowledgements}

We would like to express our gratitude to M.-G. Porquet and the SIDONIE facility of the CSNSM for providing the ${ }^{174} \mathrm{Yb}$ targets. This work is supported by the CNRS program PACEN/GEDEPEON and the EURATOM programs EFNUDAT (European Facilities for Nuclear Data Measurements) contract $n^{\circ}$ FP6-036434 and ANDES (Accurate Nuclear Data for nuclear Energy Sustainability) contract $n^{\circ}$ FP7-249671. 
EPJ Web of Conferences

\section{References}

1. J.D. Cramer, H.C. Britt, Nucl. Sci. Eng. 41, 177 (1970)

2. A. J. Koning, S. Hilaire et al., User Manual TALYS, www.talys.eu

3. G. Kessedjian et al., Phys. Lett. B 692, 297 (2010)

4. V, Weisskopf end D. H. Ewing, Phys. Rev. 57, 472 (1940)

5. J.E.Escher and F.S.Dietrich, Phys. Rev. C 81, 024612 (2010)

6. R.L.Macklin et al., Nucl Sci. Eng. 95, 189 (1987)

7. K. Wisshak et al., Phys. Rev. C 73, 015807 (2006)

8. M.V. Bokhovko et al., Vop. At. Nauki i Tekhn., Ser. Yadernye Konstanty, 44 (1985)

9. K. Wisshak et al., Phys. Rev. C 61, 065801 (2000)

10. S. Boyer et al., Nucl. Phys. A 775, 175 (2006)

11. J. N. Wilson et al., Nucl. Instr. Meth. A 511, 388 (2003)

12. B. B. Back et al., Phys. Rev. C, vol. 9, no. 5, pages 1924-1947 (1974)

13. W. Younes et al., Phys. Rev. C, vol. 67, no. 2, page 024610 (2003)

14. W. Andrejtscheff, Nucl. Phys. A 226, 142 (1974)

15. J.E. Escher, et al., CNR*09, EPJ Web of Conferences, Volume 2, vol. 20, page 6001 (2010)

16. J. Taieb. Int. Jour. of Mod. Phys. E, vol. 18, page 767, 2009. 